Hegade VS, Kendrick SF, Dobbins RL, Miller SR, Thompson D, Richards D, Storey J, Dukes GE, Corrigan M, Oude-Elferink RP, Beuers U, Hirschfield GM, Jones DEJ.

Effect of ileal bile acid transporter inhibitor GSK2330672 on pruritus in primary biliary cholangitis: a double-blind, randomised, placebo-controlled, crossover, phase 2a study.

The Lancet 2017

DOI: https://doi.org/10.1016/S0140-6736(17)30319-7

\section{Copyright:}

(C) 2017. This manuscript version is made available under the CC-BY-NC-ND 4.0 license

DOI link to article:

https://doi.org/10.1016/S0140-6736(17)30319-7

Date deposited:

$03 / 03 / 2017$

Embargo release date:

08 August 2017

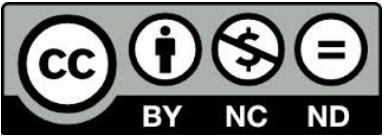

This work is licensed under a

Creative Commons Attribution-NonCommercial-NoDerivatives 4.0 International licence 


\title{
Effect of ileal bile acid transporter inhibitor GSK2330672 on pruritus in primary biliary cholangitis: a double-blind, randomised, placebo-controlled, crossover, phase 2a study
}

\author{
Authors: \\ Vinod S Hegade*1, Stuart FW Kendrick*2, Robert L Dobbins ${ }^{3}$, Sam R Miller ${ }^{2}$, Douglas \\ Thompson $^{2}$, Duncan Richards², James Storey ${ }^{2}$, George E Dukes ${ }^{3}$, Margaret Corrigan ${ }^{4}$, Ronald \\ PJ Oude Elferink ${ }^{5}$, Ulrich Beuers ${ }^{5}$, Gideon M Hirschfield ${ }^{4}$, David E Jones ${ }^{1}$
}

\begin{abstract}
Affiliations
${ }^{1}$ NIHR Newcastle Biomedical Research Centre and Institute of Cellular Medicine, Framlington Place, Newcastle University, Newcastle upon Tyne, United Kingdom

${ }^{2}$ GlaxoSmithKline, Stevenage, United Kingdom.

${ }^{3}$ GlaxoSmithKline, Research Triangle Park, NC, United States

${ }^{4}$ University of Birmingham and NIHR Birmingham Liver Biomedical Research Unit, Birmingham, United Kingdom
\end{abstract}

${ }^{5}$ Tytgat Institute for Liver and Intestinal Research, Department of Gastroenterology and Hepatology, Academic Medical Center, University of Amsterdam, Amsterdam, The Netherlands

* These authors contributed equally to the manuscript 


\section{Corresponding Author}

Dr Vinod S Hegade

NIHR Newcastle Biomedical Research Centre

Institute of Cellular Medicine

Faculty of Medical Sciences

Newcastle University

Newcastle upon Tyne NE2 4HH

United Kingdom

Phone: +441912088782 E-mail: Vinod.hegade@ncl.ac.uk 


\section{Summary}

Background: Up to $70 \%$ of patients with primary biliary cholangitis develop pruritus (itch) during the course of their disease. Treatment of pruritus in primary biliary cholangitis is challenging and novel therapies are needed. Ursodeoxycholic acid, the standard first-line treatment for primary biliary cholangitis, is largely ineffective for pruritus. We investigated the efficacy and safety of GSK2330672, a selective inhibitor of human ileal bile acid transporter (IBAT), in patients with primary biliary cholangitis with pruritus.

Methods: We conducted this phase 2a, double-blind, randomised, placebo-controlled, crossover trial in two UK medical centres. Following 2 weeks of open placebo run-in, patients were randomly assigned in a 1:1 ratio with a block size of 4 to receive GSK2330672 or placebo twice daily during two consecutive 14-day treatment periods in a crossover sequence. The treatment periods were followed by a 14-day single-blinded placebo follow-up period. The primary endpoints were safety of GSK2330672, assessed using clinical and laboratory parameters, and tolerability as rated by the Gastrointestinal Symptom Rating Scale. The secondary endpoints were changes in pruritus scores measured using the 0 to 10 numerical rating scale (NRS), primary biliary cholangitis-40 (PBC-40) itch domain score and 5-D itch scale, changes in serum total bile acids and 7 alpha hydroxy-4-cholesten-3-one (C4), and changes in the pharmacokinetic parameters of ursodeoxycholic acid and its conjugates. The trial was registered with ClinicalTrials.gov, number NCT01899703.

Findings: Between March 10, 2014, and Oct 7, 2015, we enrolled 22 patients. 11 patients were assigned to receive intervention followed by placebo (sequence 1), and 11 patients were assigned to receive placebo followed by intervention (sequence 2). One patient assigned to sequence 2 withdrew consent prior to receiving randomised therapy. One patient did not attend the placebo follow-up period, but was included in the final analysis. GSK2330672 treatment for 14 days was safe with no serious adverse events reported. Diarrhoea was the most frequent 
adverse event during treatment with GSK2330672 (seven with GSK2330672 vs one with placebo) and headache was the most frequent adverse event during treatment with placebo (seven with placebo vs six with GSK2330672). After GSK2330672 treatment, the percentage changes from baseline itch scores were $-57 \%(95 \% \mathrm{CI}-73$ to $-42, \mathrm{p}<0 \cdot 0001)$ in the NRS, $31 \%(-42$ to $-20, \mathrm{p}<0 \cdot 0001)$ in the PBC-40 itch domain and $-35 \%(-45$ to $-25, \mathrm{p}<0 \cdot 0001)$ in the 5-D itch scale. GSK2330672 produced significantly greater reduction from baseline than the double-blind placebo in the NRS $(-23 \%, 95 \% \mathrm{CI}-45$ to $-1 ; \mathrm{p}=0 \cdot 037)$, PBC-40 itch domain, $(-14 \%,-26$ to $-1 ; p=0 \cdot 034)$, and $5-\mathrm{D}$ itch scale $(-20 \%,-34$ to $-7 ; p=0 \cdot 0045)$. After GSK2330672 treatment, serum total bile acid concentrations declined by 50\% (95\% CI -37 to $-61, \mathrm{p}<0 \cdot 0001$ ) from 30 to $15 \mu \mathrm{M}$, with a significant $3 \cdot 1$-times increase (95\% CI $2 \cdot 4$ to $4 \cdot 0$, $\mathrm{p}<0 \cdot 0001$ ) in serum $\mathrm{C} 4$ concentrations from $7 \cdot 9$ to $24 \cdot 7 \mathrm{ng} / \mathrm{mL}$.

Interpretation: In patients with primary biliary cholangitis with pruritus, 14 days of ileal bile acid transporter inhibition by GSK2330672 was generally well tolerated without serious adverse events, and demonstrated efficacy in reducing pruritus severity. GSK2330672 has the potential to be a significant and novel advance for the treatment of pruritus in primary biliary cholangitis. Diarrhoea, the most common adverse event associated with GSK2330672 treatment, might limit the long-term use of this drug.

Funding: GlaxoSmithKline and National Institute for Health Research. 


\section{Research in context}

\section{Evidence before this study:}

Chronic pruritus is a common symptom associated with cholestatic liver diseases and, along with fatigue, accounts for the greatest burden of symptoms in patients with primary biliary cholangitis. Up to $70 \%$ of patients develop pruritus at some point during the course of their disease and many patients describe persistent or severe pruritus which can be debilitating. This form of pruritus goes beyond cutaneous irritation, with secondary effects through sleep deprivation, daytime somnolence, fatigue, depression, and even on occasions, suicidal ideation. Overall, patients with primary biliary cholangitis and pruritus have a poor quality of life reflecting the limitations of current approaches to treatment. We searched PubMed for clinical studies published in English between Jan 1, 1950, and Sept 1, 2016, with terms "PBC", "cholestasis", "pruritus", or "itch" and "bile acids", "bile salts", "IBAT", or "ASBT". Ursodeoxycholic acid, the standard of care for primary biliary cholangitis, has no substantial effect on pruritus and obeticholic acid, a second-line therapy, can actually worsen it. The four classes of available, guideline recommended drugs for pruritus include bile acid sequestrants (cholestyramine), pregnane $\mathrm{X}$ receptor agonists (rifampicin), opioid antagonists (naltrexone), and selective serotonin reuptake inhibitors (sertraline). However, the strength of evidence for these drugs is poor to moderate with only rifampicin and naltrexone ever studied in methodologically robust randomised controlled trials. Furthermore, rifampicin is associated with a risk of liver injury necessitating regular monitoring of liver enzymes, and naltrexone is associated with unpleasant symptoms of the opioid withdrawal syndrome. Cholestyramine remains the only licensed treatment but is poorly tolerated by many patients due to its unpleasant taste and texture, resulting in poor therapy adherence. Another bile acid sequestrant colesevelam has better tolerability but the only randomised controlled trial so far did not show that it was more effective than placebo in treating pruritus in primary biliary cholangitis. 


\section{Added value of this study}

This study is a first-in-class, randomised, placebo-controlled trial of an ileal bile acid transporter (IBAT) inhibitor to treat pruritus in patients with primary biliary cholangitis. Using three different complementary patient-reported outcome measurements, GSK2330672 showed greater effects than placebo in reducing itch intensity, as well as night-time sleep interference and daytime fatigue. GSK2330672 significantly decreased serum activity of autotaxin, which forms lysophosphatidic acid, a novel proposed pruritogen in cholestasis shedding further light on the potential mechanisms for cholestatic pruritus and the actions of the drug. In addition to improving symptoms of primary biliary cholangitis, this study has shown that pharmacological inhibition of IBAT can be used as a therapeutic strategy to decrease the circulating bile acid pool in cholestatic patients. GSK2330672 decreased serum conjugated bile acids and resulted in $\sim 50 \%$ decrease in total bile acid concentrations.

\section{Implication of all the available evidence}

Because of the great burden of pruritus in patients and the limitations of treatment options, there is a real unmet need for effective anti-pruritic therapies in primary biliary cholangitis. IBAT inhibitors are a novel class of drugs that have shown therapeutic potential in cholestasis. The results of our early phase randomised controlled trial in conjunction with previous experimental evidence and healthy volunteer trials provide support to further investigate antipruritic effect of IBAT inhibitor drugs in larger and longer-term studies of patients with primary biliary cholangitis. 


\section{Introduction}

Primary Biliary Cholangitis (previously cirrhosis, $\mathrm{PBC})^{1}$ is a chronic autoimmune liver disease characterised by progressive cholestasis. Pruritus (itch) is a frequent and troublesome symptom in $\mathrm{PBC}$ patients. The natural history of $\mathrm{PBC}$ suggests that $60-70 \%$ of patients develop pruritus at some point during the disease process..$^{2-4}$ The pathogenesis of cholestatic pruritus is complex and a number of putative pruritogens have been proposed. ${ }^{5}$ The use of ursodeoxycholic acid (UDCA), the current standard of care in PBC, has improved outcomes in PBC but has not been shown to improve pruritus. ${ }^{6}$

The bile acid sequestrant cholestyramine is often given to treat pruritus, but its effectiveness in practice is limited. Despite its modest evidence base, and poor tolerability profile, it is the only US Food and drug Administration (FDA)-approved therapy for cholestatic pruritus and is recommended by both the American and European practice guidelines as the first-line agent. ${ }^{7}$ ${ }^{8}$ Colesevelam is a better tolerated bile acid sequestrant, but was not efficacious in the only randomised controlled trial reported so far. ${ }^{9}$ Other drug therapies (rifampicin, naltrexone, and sertraline), although recommended by the scientific guidelines, ${ }^{7,8}$ are not actually licensed for treating cholestatic pruritus. Moreover, they have the disadvantage of needing regular monitoring due to risk of liver injury and other limiting adverse effects. This is particularly an issue with rifampicin, the most widely used second-line therapy, which has well reported hepatotoxicity. ${ }^{10}$ In clinical practice, response rates less than $50 \%$ are common for most of the guideline recommended drugs ${ }^{11}$ and despite their step-wise use, many patients report refractory itch, which can only be treated by invasive (usually temporary) treatments such as nasobiliary drainage $^{12}$ or liver transplantation (the only definitive cure). Therefore, effective anti-pruritic drug therapy is an unmet clinical need in primary biliary cholangitis and other cholestatic liver diseases. $^{13}$ 
The paucity of effective anti-pruritic therapies in primary biliary cholangitis is probably compounded by the fact that the key emerging second-line disease modifying agent, obeticholic acid, which has been licensed by the FDA and European Medicines Agency, is associated with an increased frequency and severity of pruritus. ${ }^{14,15}$ Many other bile acid-based therapies in primary biliary cholangitis that are in development 16 might also be associated with pruritus. Therefore, effective pruritus management in primary biliary cholangitis is likely to become increasingly important and challenging and new approaches are needed.

Ileal bile acid transporter (IBAT, also called apical sodium-dependent bile acid transporter [ASBT]) is an integral brush border membrane glycoprotein mainly expressed in the distal ileum. ${ }^{17,} 18$ The main physiological function of IBAT is reabsorption of bile acids and maintenance of their enterohepatic circulation. In cholestatic liver disease, ileal bile acid absorption is increased ${ }^{19,20}$ and inhibiting ileal bile acid transport was proposed to prevent inappropriate conservation of bile acids. ${ }^{21}$ Using an IBAT inhibitor to reduce bile acid reabsorption and modulate the bile acid pool in the systemic circulation is an unexplored therapeutic strategy in primary biliary cholangitis, a condition in which retention of toxic hydrophobic bile acids is postulated to play a key pathogenetic role.

To date, published reports of IBAT inhibitor drugs include a study in healthy people (A4250),22 two reports in animal models of cholestasis (A4250 and SC435) $)^{23,24}$ and a report of lopixibat chloride (formerly LUM001) in patients with primary biliary cholangitis and pruritus. ${ }^{25}$ GSK 2330672 is a highly potent, soluble, minimally absorbed, selective inhibitor of the human IBAT. It has been assessed in animal models of type 2 diabetes mellitus (T2DM) and an early phase trial of T2DM patients. ${ }^{26,27}$ In two phase 1 studies (59 healthy volunteers) it was well tolerated with a good safety profile at a dose range of $0 \cdot 1$ to $90 \mathrm{mg}$ (unpublished data from clinical trial NCT01416324 and NCT01607385). 
Here we report the first randomised, placebo controlled, double-blind, crossover, phase 2 trial of an IBAT inhibitor in people with primary biliary cholangitis and pruritus. We postulated that GSK2330672 would interrupt enterohepatic circulation of bile acids and exert therapeutic benefit on pruritus associated with primary biliary cholangitis. 


\section{Methods}

\section{Study design and patients}

BAT117213 was a phase 2a randomised, double-blind, placebo-controlled study to assess the safety, tolerability, pharmacokinetics, and pharmacodynamics of repeat doses of GSK2330672 in patients with primary biliary cholangitis and symptoms of pruritus (figure 1). This study was done at two centres in the UK: Newcastle Upon Tyne Hospitals NHS Foundation Trust, Newcastle upon Tyne, and University Hospitals Birmingham NHS Foundation Trust, Birmingham, in collaboration with the UK-PBC Consortium. ${ }^{28}$ The National Research Ethics Service Committee North East and Sunderland (REC reference 13/NE/0290) and the Medicine and Healthcare products Regulatory Agency approved all versions of the study protocol which is available online. ${ }^{28}$ All recruitment sites obtained approval from their respective hospital Research and Development (R\&D) departments before screening patients. All participants provided written informed consent before enrolment. The trial was done in accordance with the International Conference on Harmonisation Good Clinical Practice guidelines and the Declaration of Helsinki. ${ }^{29}$ The trial protocol is available online. ${ }^{28}$

Patients were eligible for inclusion in the trial if they were aged 18-75 years, had proven or likely primary biliary cholangitis with ongoing pruritus, were on stable doses of UDCA for more than 8 weeks at the time of screening, and had serum alkaline phosphatase value no more than 10 times the upper limit of normal. Exclusion criteria are shown in the appendix.

Eligible volunteers entered a 2 week open placebo run-in period followed by randomised, blinded, two-period crossover treatment periods (sequence 1 and sequence 2) of 14 days each during which patients received either GSK2330672 or placebo twice daily. Sequence 1 was GSK2330672 followed by placebo and sequence 2 was placebo followed by GSK2330672. There was no washout period between two treatment periods. Patients then entered 14 days of follow-up during which they received blinded placebo treatment. When taking GSK2330672 
(or matching placebo), participants received $45 \mathrm{mg}$ twice per day on days $1-3$, and were then asked to increase the dose to $90 \mathrm{mg}$ twice daily on days 4-14.

\section{Outcomes}

The primary objective of the study was to investigate the safety and tolerability of oral GSK2330672 when administered for 14 days to patients with primary biliary cholangitis with pruritus. The secondary objectives were to investigate the effects of oral GSK2330672 on participants' experience of pruritus, study the effect of the drug on serum total bile acids and 7 alpha hydroxy-4- cholesten-3-one (C4), a serum marker of bile acid synthesis, and investigate the steady-state pharmacokinetic parameters of UDCA and its taurine and glycine conjugates. Exploratory endpoints included changes in the concentrations of serum liver biochemistry, bile acid species, autotaxin (ATX) activity, and fibroblast growth factor (FGF19; appendix).

\section{Randomisation and masking}

A single randomisation schedule for all sites was generated using a dedicated randomisation creation and publishing tool for GlaxoSmithKline (GSK) studies (RandAll) by the GSK statistician. Randomisation numbers were allocated in a 1:1 ratio to sequence 1 or sequence 2 with a block size of four. Randomisation numbers were allocated to participants by site staff .Patients, investigators, clinical trial site staff, and sponsor staff directly involved with the study were masked to treatment sequence assignment throughout the study.

\section{Statistical analysis}

The study was designed to estimate the effect of GSK2330672 (when co-administered with UDCA) relative to placebo on pruritus and the pharmacokinetics of UDCA. Due to the early clinical and exploratory nature of the study, no formal hypothesis testing was planned and the sample size was based on feasibility with consideration of efficacy (using pruritus $0-10$ numerical rating scale $[\mathrm{NRS}]$ ) and potential pharmacokinetics interaction between 
GSK2330672 and UDCA. An initial sample size of 40 participants was estimated to be sufficient for both efficacy and pharmacokinetics based on the assumption that GSK2330672 was at least as efficacious as rifampicin and the SD was similar to that reported in trials of other anti-pruritic drugs. ${ }^{30,31}$ Given the uncertainties associated with sample size assumptions, two interim analyses were done to assess futility and determine if sample size re-estimation was necessary. Data from the pruritus 0-10 NRS were reviewed by a non-blinded Interim Review Committee (composed of people not directly involved in study conduct). The first interim analysis was done after 11 patients had completed the treatment period, and a second interim analysis was done after 19 patients had completed the treatment period, at which point the final target sample size was reduced (appendix). The study was closed for recruitment after 22 people were randomly assigned.

To summarise the daily pruritus $0-10$ point NRS during the placebo run-in period and each treatment period for each individual patient, we calculated trimmed means of weekly itch scores. Trimmed means removed the highest and lowest daily score (an average of the morning and evening scores) to provide more robust summary not influenced by potential outlying values. For statistical analysis we used the second week of each period to provide an analytical washout (i.e., 7 days between the analysed periods to allow treatment effects to stabilise). The efficacy endpoint analysis used a mixed effects model with fixed effect terms for treatment period and sequence, with participants treated as a random effect in the model. Baseline (placebo run-in) results were included in the model as an additional period. Point estimates and their associated 95\% CI and $\mathrm{p}$ value were constructed for the mean differences of interest in pruritus scores (i.e., changes from baseline on each treatment and between double-blind GSK2330672 and placebo). The statistical analysis of pruritus scores in PBC-40 and 5-D itch was similarly done (appendix). Data on pharmacokinetics parameters, bile acids, and biomarkers were log transformed for analysis, and results are reported as percentage changes 
or ratios. Although not formally a hypothesis-testing study, two-sided p values less than $0 \cdot 05$ were considered statistically significant. All analyses were done using SAS, version 9.2 or greater (SAS Institute, Cary, NC, USA). This trial is registered on ClinicalTrials.gov, number NCT01899703.

\section{Role of the funding source}

The sponsor and main study funder (GlaxoSmithKline; GSK) was involved in the study design, data analysis, and data interpretation with inputs from VSH, DEJ, and GMH. The NIHR Newcastle Biomedical Research Centre provided additional funding but had no role in study design, data collection, analysis, or interpretation. The trial was supervised by the research and development department of the Newcastle Upon Tyne Hospitals NHS Foundation Trust and data collection occurred at the clinical trial units of the study centres. The corresponding author (VSH) had access to the raw data, did analysis of primary and secondary outcomes with SRM and DT (GSK statisticians) and wrote the study report. All authors had access to the data, reviewed the manuscript, and share final responsibility for the decision to submit for publication. 


\section{Results}

Between March 10, 2014, and Oct 7, 2015, we enrolled and randomised 22 patients. 21 patients completed all the planned study procedures as per protocol. One patient was withdrawn from the study due to withdrawal of consent in the placebo run-in period (figure 2). The safety population therefore comprised a total of the 22 randomly assigned patients, while the analysis population comprised 21 patients who completed all the planned study procedures as per protocol (although one patient did not attend the full follow-up period). 19 of 21 patients were taking UDCA during the study period at the guideline recommended dose. The baseline demographic and clinical characteristics of the participants are shown in table 1. A summary of the frequency of use of anti-pruritic treatments prior to the start of the study is provided in the appendix ( $\mathrm{p}$ 15). As per the study protocol, use of these drugs was stopped at the study entry.

All patients in the active treatment period started with GSK2330672 at a dose of $45 \mathrm{mg}$ twice per day for 3 days and successfully increased to $90 \mathrm{mg}$ twice per day on days $4-14$. During the study there were no reports of serious adverse events. There were no clinically significant changes in vital signs, laboratory values or ECG parameters, and no positive faecal occult blood tests were reported. There were no reports of liver toxicity and no significant changes were seen in serum total bilirubin, alkaline phosphatase, or other liver enzymes during the study period (appendix p 16).

Overall, GSK2330672 was well tolerated. A summary of all adverse events reported for more than one participant during any treatment period (irrespective of sequence) is given in table 2 . The frequency of any adverse events was similar in active drug and placebo treatment periods ( $81 \%$ vs $81 \%$ ). The most common adverse event observed during the study was headache, reported by 14 of 22 participants. 16 patients reported adverse events related to the gastrointestinal system. The most common GSK2330672-related adverse event was diarrhoea, 
reported by seven participants, with five rating the severity as mild (lasting up to 4 days with no or minimal effect on daily life). The frequency of diarrhoea reported during GSK2330672 treatment was significantly higher compared with placebo (seven patients during GSK2330672 treatment $v s$ one patient during placebo treatment, $\mathrm{p}=0 \cdot 0391$, post-hoc mid-p McNemar test). No patient discontinued the drug or had their dose decreased secondary to diarrhoea. Two adverse events (diarrhoea, abdominal distension) reported during GSK2330672 treatment and one adverse event of upper abdominal pain reported during placebo treatment were considered to be severe.

After GSK2330672 treatment, changes from baseline itch scores were: -57\% (95\% CI -73 to $42, \mathrm{p}<0 \cdot 0001)$ for NRS, $-30 \%(-42$ to $-20, \mathrm{p}<0 \cdot 0001)$ for PBC-40 itch domain, and $-35 \%(-$ 45 to $-25, \mathrm{p}<0 \cdot 0001$ ) for 5-D itch scale (figure 3). GSK2330672 reduced itch intensity significantly more than the double-blind placebo in all three scales: $-23 \%(-1$ to $-45, \mathrm{p}=0$ 0374) for NRS, $-14 \%(-1$ to $-26, p=0-0335)$ for PBC-40 itch domain, and $-20 \%(-7$ to -34 , $\mathrm{p}=0 \cdot 0045)$ for $5-\mathrm{D}$ itch.

For individual patients, changes in the weekly trimmed mean of their itch intensity score are shown in the appendix. Overall, the mean NRS itch intensity score decreased significantly from baseline after GSK2330672 treatment period (appendix p 17) and the reduction was significant in both the sequences of treatment (appendix p 18). In the NRS, itch was also assessed for worst itch, bothersome itch, and sleep interference. Significant reductions were seen in these scores following GSK2330672 (appendix p 19).

GSK2330672 treatment was also associated with significant reductions in the mean PBC-40 itch domain and 5-D itch score (appendix p 20). The mean decrease from baseline in 5-D itch score after GSK2330672 treatment was significant in both sequences of treatment, whereas the decrease in PBC-40 itch domain score was only significant in sequence 2. Adjusting for 
sequence and period, the mean $\mathrm{PBC}-40$ itch domain and 5-D itch scores following GSK2330672 treatment were significantly lower than placebo.

Analysis of other domains of PBC-40 showed a significantly greater reduction in the fatigue domain score after GSK2330672 treatment compared with placebo $(-9 \%, 95 \%$ CI -3 to -16 ; $p=0 \cdot 0033$ ). No significant changes were apparent for other domains of PBC-40 (appendix pp 21-22). All five domains of the 5-D itch scale, including the disability domain, showed significantly lower scores after GSK2330672 compared with placebo (appendix pp 23-24).

Serum total bile acid concentrations changed from baseline with a 50\% decrease $(95 \%$ CI -37 to $-61, \mathrm{p}<0 \cdot 0001)$ after GSK2330672 treatment compared with a $12 \%$ increase $(-12$ to 42 , $\mathrm{p}=0 \cdot 3540)$ after placebo. The changes in serum total bile acid concentrations following GSK2330672 were significant when compared with baseline and placebo (figure 4A) and the changes were reversed within 2 weeks of stopping GSK2330672. Conversely, faecal total bile acid showed a mean $36 \%$ increase after GSK2330672 (95\% CI -1 to 85) compared with 16\% decrease after placebo ( -40 to 15$)$.

Serum concentrations of conjugated bile acids decreased after GSK2330672 compared with baseline (appendix p 25), with the largest percentage reductions observed in taurocholate ($74 \% ; 95 \%$ CI -53 to $-86, \mathrm{p}<0 \cdot 0001)$, glycocholate $(-64 \% ;-23$ to $-83, \mathrm{p}=0 \cdot 0099)$, and taurochenodeoxycholate $(-58 \% ;-32$ to $-74, p=0 \cdot 0007)$. In contrast, unconjugated primary bile acids cholate $(-13 \% ;-60$ to $86, p=0 \cdot 7040)$ and chenodeoxycholate $(-4 \% ;-34$ to $38, p=0$ - 8049) did not change significantly after GSK2330672 (appendix p 25). A significant increase in serum UDCA $(57 \% ; 15$ to $116, \mathrm{p}=0 \cdot 0062)$ and a marginal increase in serum deoxycholate $(11 \% ;-1$ to $23 \%, p=0 \cdot 0619)$ were observed after GSK2330672 (appendix p 25). No 
significant changes from baseline were seen in any bile acid species after placebo treatment (appendix pp 27-29).

There was a significant $3 \cdot 1$-times increase in serum $\mathrm{C} 4$ concentration from baseline after GSK2330672 treatment (95\% CI $2 \cdot 4$ to $4 \cdot 0, \mathrm{p}<0 \cdot 0001$; figure 4B) and the increase was seen in both sequences of treatment. No significant changes in $\mathrm{C} 4$ were seen after placebo treatment. Significant decreases compared with baseline were seen in serum ATX activity ($11 \%, 95 \% \mathrm{CI}-3$ to $-19 ; \mathrm{p}=0 \cdot 0070)$ and FGF19 concentration $(-78 \%,-60$ to $-88 ; \mathrm{p}<0 \cdot 0001)$ following GSK2330672 treatment, but not after placebo (appendix).

GSK2330672 is designed as a minimally absorbable agent with minimal systemic exposure. Eight of 22 participants had measurable plasma concentrations of GSK2330672 and the peak concentration $(5 \cdot 33 \mathrm{ng} / \mathrm{mL}$, achieved at $2 \mathrm{~h}$ post-dose in one patient) is consistent with minimal systemic exposure. All 14 patients who provided faecal samples for drug analysis had detectable GSK2330672 in the faeces. No GSK2330672-related metabolites were detected in plasma or urine. Comparing co-administration of UDCA with GSK2330672 or with placebo showed $90 \%$ CIs for UDCA maximum concentration (Cmax) and area under curve (AUC) ratios within conventional bioequivalence limits of $0 \cdot 8-1 \cdot 25$ but reduced exposure in the metabolites tauroursodeoxycholic acid (TUDCA) and glyco ursodeoxycholic acid (GUDCA; appendix). 


\section{Discussion}

This trial of GSK2330672 shows safety and efficacy of a first-in-class, novel agent in treating pruritus associated with primary biliary cholangitis. Despite the substantial symptom burden and need for better anti-pruritic drugs, little progress in developing new treatments for pruritus in primary biliary cholangitis has been made. We have attempted to fill this treatment gap with the first randomised, placebo-controlled, crossover trial of an IBAT inhibitor drug in patients with primary biliary cholangitis and clinically significant pruritus. Our results suggest that interrupting enterohepatic circulation of bile acids by inhibiting IBAT with GSK2330672 improves pruritus in patients with primary biliary cholangitis.

We found that GSK2330672 at 45-90 mg dose, given twice per day for 2 weeks in patients with primary biliary cholangitis, was safe and generally well tolerated. No serious adverse events and no clinically significant abnormality related to haematology, clinical chemistry, or ECG was reported following GSK2330672 treatment. Diarrhoea was the most frequent adverse event associated with GSK2330672, which is in concordance with previous reports of IBAT inhibition in healthy volunteers ${ }^{22}$ and in patients with T2DM. ${ }^{27}$ Increased bile acid load in the colon increases colonic motility and reduces colonic transit time causing diarrhoea. ${ }^{32-34}$ In our study, severity of diarrhoea was mild to moderate and no patients discontinued GSK2330672 or had their dose decreased. Taken together, the safety and tolerability profile of GSK2330672 in patients seen in our study does not preclude further clinical investigation of the drug to treat patients with primary biliary cholangitis; however, gastrointestinal disturbance might ultimately limit the usefulness of this agent in practice.

The key finding of this study is that GSK2330672 was significantly more efficacious than placebo in improving itch intensity. This was substantiated by decreases in pruritus scores measured by three different tools of itch measurement. GSK2330672 treatment was associated with improvement in pruritus and the changes from baseline were significant regardless of the 
dosing sequence. Notably, pruritus scores improved within the first week of GSK2330672, continued to decrease through 2 weeks of treatment, and returned towards baseline on switch to blinded placebo. The improvement in pruritus severity seen in the placebo phases was in keeping with other trials of anti-pruritic agents in primary biliary cholangitis and was significantly exceeded by that seen in the active drug phases.

Despite the differences in the results between intervention and placebo treatment, the magnitudes of the effect could be underestimated because the crossover study design lacked a washout between treatment periods. Incorporation of the analytical washout mitigates this somewhat, but placebo responses and carry-over effects still resulted in a sequence effect that influenced the magnitude of response depending on the order of treatment. Comparing the GSK2330672 period with the run-in open placebo period avoids the sequence effect and gives an alternative estimate of the magnitude of effect, although this might be an overestimation due to the unblinded placebo run-in being used to form this comparison. Nevertheless, individual patient responses (appendix) clearly showed rapid improvement of pruritus during GSK2330672 treatment and greater response was observed in patients with higher baseline itch intensity than in those with lower itch intensity. GSK2330672 also decreased sleep interference score, disability (5-D itch scale), and fatigue (PBC-40 scale) domain scores agreeing that the treatment had clinically meaningful effect on the symptom complex associated with primary biliary cholangitis. The study provides early evidence of effect in this debilitating symptomatic condition, but treatment duration was not sufficient to assess durability of benefit. This study had few patients and was not designed to deliver definitive conclusions on superiority of study drug over placebo. The interim analyses might have introduced some statistical bias in the estimated effect sizes, though as the interim analyses primarily assessed lack of effect we do not anticipate that this bias would greatly affect the results. Therefore, the efficacy of GSK2330672 on pruritus needs to be substantiated in larger studies of longer duration. 
The evidence for the role of bile acids in the development of pruritus in primary biliary cholangitis has been equivocal, and the topic has been controversial. ${ }^{35} \mathrm{~A}$ strong association between plasma bile acid concentration and the severity of itch has never been recorded in cholestatic patients ${ }^{35}$ but other evidence has linked bile acid-mediated cholestatic pruritus via TGR5 receptors. ${ }^{36,} 37$ In this study, GSK2330672 treatment had substantial effect on the circulating bile acid pool, as shown by $50 \%$ decrease in serum total bile acid and decreased serum concentration of all taurine and glycine conjugated primary bile acids. These findings are consistent with a preferential effect of GSK2330672 on ileal reuptake of conjugated bile acids. However, we cannot exclude the possibility that pruritogens other than bile acids are also transported via IBAT. Cholesterol $7 \alpha$-hydroxylase (CYP7A1), the rate limiting enzyme in hepatic bile acid synthesis, is regulated by farnesoid X receptor (FXR) and FGF19. Following GSK2330672 treatment we observed a significant 3-times increase in serum C4, a surrogate marker for hepatic CYP7A1 enzymatic activity. ${ }^{38}$ Fasting C4 has been shown to provide a good measure of the overall flux through the bile acid synthetic pathway and a reliable assessment of the degree of IBAT inhibition. ${ }^{27}$ Taken together, these findings suggest substantial target (i.e., IBAT) engagement and inhibition by GSK2330672. However, post-hoc correlation analyses estimated correlation coefficients between circulating biomarkers and pruritus scores to be less than $0 \cdot 4$, indicating that these markers are not highly associated with efficacy at an individual patient level after a short period of treatment.

Lopixibat chloride (maralixibat or LUM001), an IBAT inhibitor, has been studied in a phase 2 RCT as a novel treatment of pruritus in patients with primary biliary cholangitis (CLARITY study). ${ }^{25}$ Although the itch scores decreased from baseline in both lopixibat and placebo groups, lopixibat was not shown to be more efficacious than placebo in reducing pruritus. The negative result is likely to be, in part, due to a lower bile acid depleting effect of lopixibat. In our study, the effects of GSK2330672 on both total bile acid and C4 were more marked than 
those reported with lopixibat; a finding which might explain the difference in clinical efficacy of the two agents.

The significant decrease in serum FGF19 concentration observed in our study is consistent with decreased ileal FXR activation following IBAT inhibition. Decreased circulating concentrations of FGF19 result in decreased inhibition of hepatic bile acid synthesis, as reflected by increased serum C4 concentration. In this regard, the effect of GSK2330672 on FGF19 is different to that of other agents proposed for primary biliary cholangitis treatment. Obeticholic acid is a strong ileal FXR activator that increases FGF19 concentration and reduces bile acid synthesis, and fibrates reduce bile acid synthesis despite reduction in FGF19. The long-term use of an IBAT inhibitor is likely to maintain suppressed FGF19 and therefore, the effect of resulting upregulated bile acid synthesis on cholestasis needs careful investigation. However, the circulating bile acid pool is unlikely to expand as the loss of bile acids in faeces might exceed increased bile acid synthesis. The apparent paradox of contrasting actions of different agents in primary biliary cholangitis on FGF19 reflects that this is only an intermediate step in one pathway reducing bile acid concentration. If reduction in bile acid is the crucial pathway, then direct reduction through IBAT inhibition could be as valuable a mechanism as suppression of synthesis despite contrasting actions on FGF19.

Autotaxin (ATX) has been proposed as a key potential factor in the pruritogenic pathway in cholestasis. ${ }^{39,}{ }^{40} \mathrm{We}$ observed significantly reduced serum ATX activity after treatment with GSK2330672 but not after placebo. It is possible that IBAT inhibition with GSK2330672 also interrupts the ileal reabsorption of an as yet unidentified molecule that might upregulate ATX activity, ${ }^{41}$ which in turn decreases the expression or synthesis of ATX and reduces serum ATX concentration. Further work is required to probe the mechanisms involved in ATX effects of IBAT inhibitors. 
The ability of GSK2330672 to remain in the gastrointestinal tract reduces concerns of systemic toxicity and drug interactions. Notably, GSK2330672 did not have any significant interaction with UDCA absorption or recycling. UDCA is not transported via IBAT and there was a significant increase in serum UDCA concentration after GSK2330672 treatment. However, the glyco (GUDCA) and tauro (TUDCA) conjugates of UDCA are transported by IBAT and we observed a 3-4-times decrease in their serum concentration. The clinical relevance of this effect is not known. Reassuringly, we did not observe any adverse effect of GSK2330672 on the therapeutic efficacy of UDCA since the serum liver biochemistry (mainly alkaline phosphatase) did not adversely increase during the study treatment. As UDCA is the mainstay of treatment in primary biliary cholangitis the clinical implication of inhibitory effect of GSK2330672 on UDCA conjugates merits further investigation.

Overall, there were no significant changes in the liver enzymes following GSK2330672 treatment. The absence of significance might be due to the short duration of treatment used in this study. Longer treatment with GSK2330672 could be required to study the effect of IBAT inhibition on alkaline phosphatase (biochemical marker of cholestasis) and other liver enzymes.

This study was designed to efficiently gather information about potential effects of GSK2330672, which if sufficiently encouraging could be used to subsequently design a definitive trial. In this context, our study has several strengths. Firstly, this is the first crossover $\mathrm{RCT}$ of an IBAT inhibitor drug to treat pruritus in patients with primary biliary cholangitis. The crossover design of the study allowed estimation of the treatment effect in a few patients and provided a more efficient comparison of treatments than a similar sized parallel group trial. Secondly, we used patient reported outcomes to measure the treatment response objectively by using the existing validated tools (PBC-40 questionnaire and 5-D itch scale) and a novel, easyto-use electronic symptom diary. The study was not designed to make definitive conclusions 
on superiority of the study drug over placebo treatment and it is limited by the small sample size, short duration ( 2 weeks) of treatment, and the absence of a washout period, as discussed above. Therefore the efficacy of GSK2330672 on pruritus needs to be substantiated by larger studies of longer duration.

In conclusion, this phase 2 a randomised controlled trial showed that 2 weeks of treatment with an oral IBAT inhibitor GSK2330672 in patients with primary biliary cholangitis and symptoms of pruritus was safe, well tolerated, and was more efficacious than placebo in reducing the severity of pruritus. There was a significant reduction in serum total and conjugated bile acids, consistent with the postulated mechanism of efficacy, interruption of enterohepatic circulation of biliary pruritogens. Our results suggest that GSK2330672 could be a substantial advance for the treatment of pruritus in primary biliary cholangitis. Depending on its occurrence, duration, and effect in further studies, diarrhoea, the most common adverse event associated with GSK2330672, might limit the long-term use of this drug. 


\section{Contributors}

SFWK, RLD, DR, and DEJ had the original concept of this trial and developed the trial protocol. SFWK, RLD, SRM, DR, JS, GMH, and DEJ wrote or reviewed all protocol versions. DEJ and GMH were principal investigators of this study. SFWK, JS, and VSH submitted all research ethics committee applications, local research and development applications and coordinated the trial sites. VSH, SFWK, MC, GMH, and DEJ recruited the participants and were responsible for data collection. VSH, SFWK, RLD, SRM, DT, JS, GED, GMH, and DEJ participated in data analysis and interpretation. SRM and DT did the sample size calculation and analysis plan. VSH, SRM, and DT did all the statistical analysis. RPJOE and UB contributed in analysis and interpretation of bile acids, FGF19, and autotaxin data. VSH was the sub-investigator of the participating centre and wrote the manuscript for this publication. SRM, DT, and VSH were responsible for preparation of the tables and figures. All authors participated in the Article review and approved the final manuscript. VSH, GED, GMH, and DEJ are guarantors.

\section{Declaration of interests}

SFWK, RLD, SRM, DT, DR, JS, and GED are or were GlaxoSmithKline (GSK) employees. SFWK reports grants and other from GSK during the conduct of the study and outside the submitted work. RLD reports personal fees from GSK during the conduct of the study. SRM reports personal fees and other from GSK during the conduct of the study. DR reports other from GSK during the conduct of the study. RPJOE received reimbursement of costs of autotaxin, bile salts, and FGF19 measurement from Newcastle University. GMH and DEJ are investigators on the UK-PBC consortium which has received research funding from GSK. GMH attended an advisory board on primary biliary cholangitis for GSK and received travel expenses for attending data analysis meeting. VSH reports personal fees and non-financial support from GSK during the conduct of the study. MC and UB declare no competing interests. 


\section{Acknowledgments}

We thank the patients enrolled in this clinical trial and the National Institute for Health Research (NIHR) Newcastle Biomedical Research Centre (BRC) for supporting the exploratory studies associated with the study. We thank LIVErNORTH (www.livernorth.org.uk) and PBC Foundation (www.pbcfoundation.org.uk) for their ongoing support of trials in primary biliary cholangitis and their assistance in raising awareness of the trial and recruiting patients. SRM and DT acknowledge statistics and programming support from the team at Quantitative Sciences, India and GSK, Stevenage. VSH acknowledges NIHR Newcastle BRC for funding his clinical $\mathrm{PhD}$ fellowship in cholestatic pruritus. MC and GMH are supported by the NIHR Birmingham Liver Biomedical Research Unit (BRU). The clinical trial visits were done at the Newcastle Biomedicine Clinical Research Facility and the Birmingham NIHR Clinical Research Facility. This paper presents independent research and the views expressed are those of the authors and not necessarily those of the National Health Service (NHS), the NIHR, or the Department of Health. 


\section{Figure 1}

Trial Design

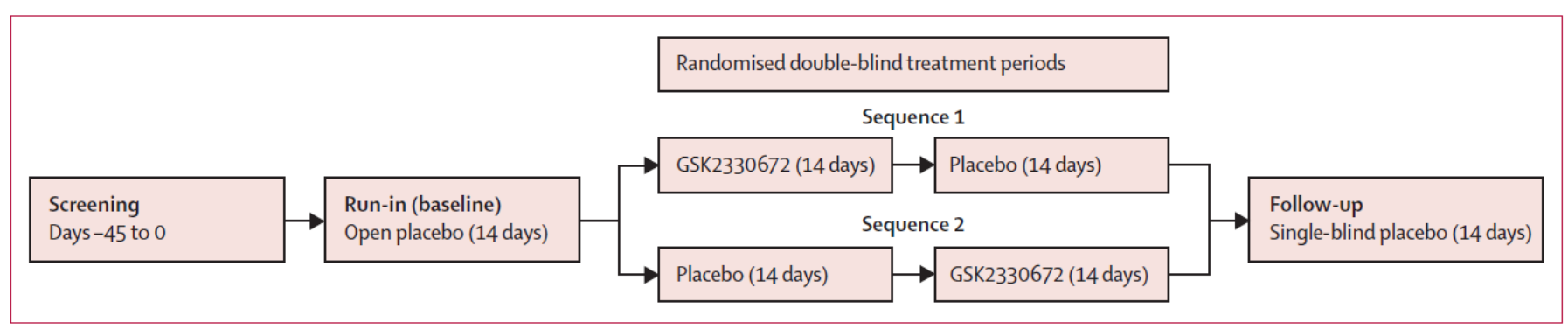




\section{Figure 2}

Trial Profile

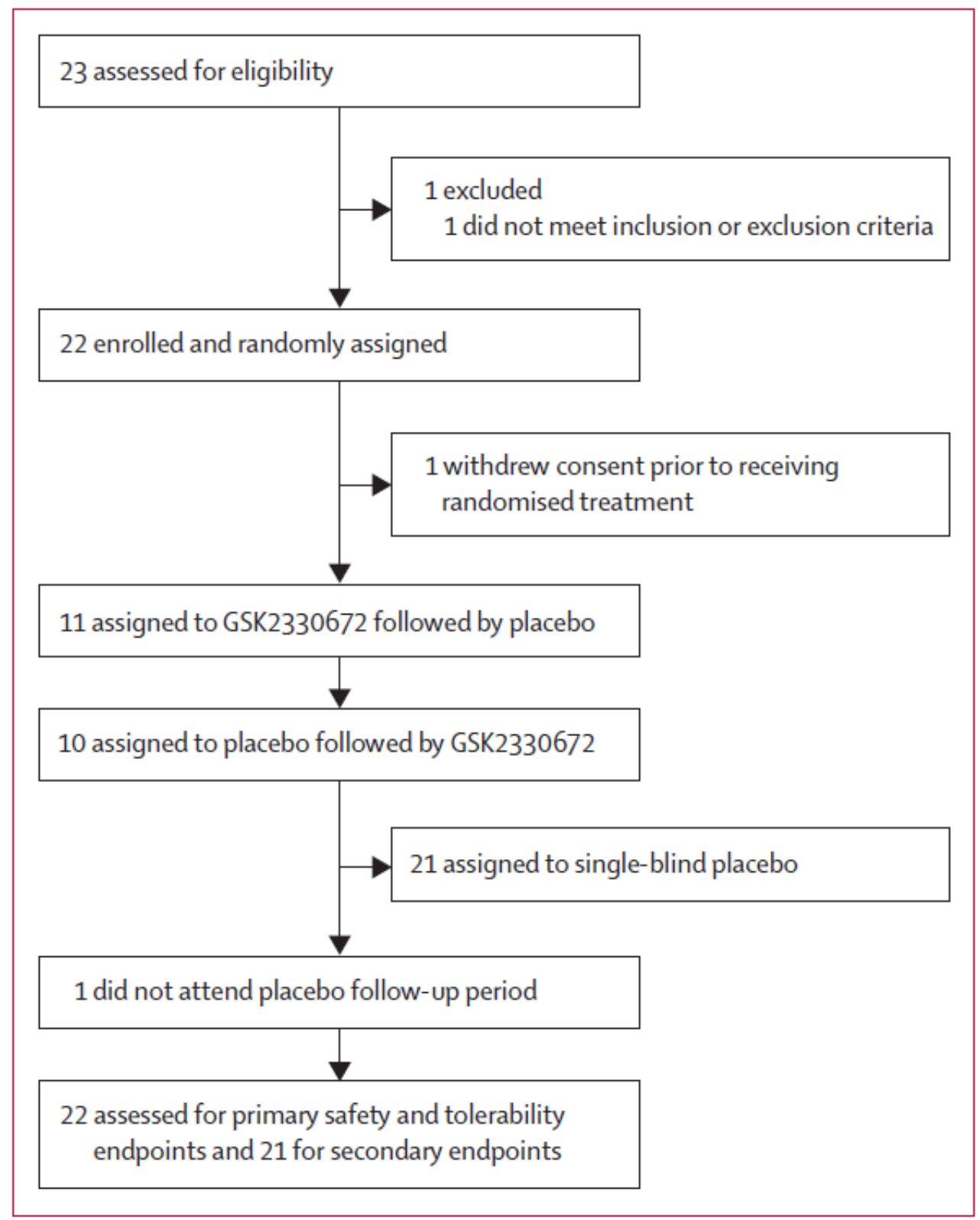




\section{Table 1}

Baseline characteristics of volunteers

\begin{tabular}{|c|c|}
\hline & $\begin{array}{l}\text { Measurement } \\
\text { at baseline }\end{array}$ \\
\hline Age (years) & $52 \cdot 9(10 \cdot 6)$ \\
\hline Female (n) & $19(86 \%)$ \\
\hline Body-mass index $\left(\mathrm{kg} / \mathrm{m}^{2}\right)$ & $27 \cdot 2(4 \cdot 9)$ \\
\hline Bodyweight $(\mathrm{kg})$ & $72 \cdot 8(13 \cdot 5)$ \\
\hline Duration of primary biliary cholangitis (years) & $5(4 \cdot 8)$ \\
\hline \multicolumn{2}{|l|}{ Race } \\
\hline White (n) & $21(95 \%)$ \\
\hline Asian: Central/South Asian (n) & $1(5 \%)$ \\
\hline \multicolumn{2}{|l|}{ Ursodeoxycholic acid (UDCA) } \\
\hline People taking UDCA during study period (n) & $19(90 \cdot 4 \%)$ \\
\hline Total daily dose during study period (mg/day) & $967(185.8)$ \\
\hline $\begin{array}{l}\text { Bodyweight adjusted daily dose during study period } \\
\text { (mg/kg/day) }\end{array}$ & $14(1 \cdot 7)$ \\
\hline \multicolumn{2}{|l|}{ Pruritus scores* } \\
\hline $\begin{array}{l}\text { Itch intensity on numerical rating scale }(\min 0 \text {, } \\
\text { max } 10) \text {, trimmed mean }\end{array}$ & $5 \cdot 33(2 \cdot 1)$ \\
\hline $\begin{array}{l}\text { Primary biliary cholangitis-40 itch domain score } \\
(\min 3, \max 15)\end{array}$ & $10 \cdot 5(3 \cdot 3)$ \\
\hline 5-D itch scale $(\min 5, \max 25)$ & $18 \cdot 7(3 \cdot 6)$ \\
\hline \multicolumn{2}{|l|}{ Laboratory markers* } \\
\hline Alkaline phosphatase (IU/L) & $264(174 \cdot 1)$ \\
\hline Gamma glutamyl transferase (IU/L) & $211(172 \cdot 6)$ \\
\hline Alanine aminotransferase (IU/L) & $59 \cdot 3(44 \cdot 8)$ \\
\hline Aspartate aminotransferase (IU/L) & $60 \cdot 8(35 \cdot 8)$ \\
\hline Total bilirubin $(\mu \mathrm{mol} / \mathrm{L})$ & $12 \cdot 2(5 \cdot 5)$ \\
\hline Total protein $(\mathrm{g} / \mathrm{L})$ & $73 \cdot 32(5 \cdot 9)$ \\
\hline Albumin $(g / L)$ & $41 \cdot 9(4 \cdot 2)$ \\
\hline Creatinine $(\mu \mathrm{mol} / \mathrm{L})$ & $65 \cdot 8(9 \cdot 1)$ \\
\hline Autotaxin activity (nmol/ml per min) & $8 \cdot 2(4 \cdot 1)$ \\
\hline $\mathrm{FGF} 19(\mathrm{pg} / \mathrm{mL})$ & $162 \cdot 9(107 \cdot 5)$ \\
\hline $\mathrm{C} 4(\mathrm{ng} / \mathrm{mL})$ & $13 \cdot 1(10 \cdot 0)$ \\
\hline Total bile acids $(\mu \mathrm{M})$ & $48 \cdot 6(68 \cdot 7)$ \\
\hline \multicolumn{2}{|c|}{$\begin{array}{l}\text { Data are shown in mean (SD) unless otherwise stated. *Baseline data at the enc } \\
\text { of placebo run-in period. }\end{array}$} \\
\hline
\end{tabular}


Table 2

Summary of adverse events

\begin{tabular}{|c|c|c|c|}
\hline & $\begin{array}{l}\text { Placebo run-in } \\
(\mathrm{n}=22), \mathrm{n}(\%)\end{array}$ & $\begin{array}{l}\text { GSK2330672 } \\
(n=21), n(\%)\end{array}$ & $\begin{array}{l}\text { Placebo } \\
(n=21), n(\%)\end{array}$ \\
\hline Participants with any adverse event & $15(68)$ & $17(81)$ & $17(81)$ \\
\hline \multicolumn{4}{|l|}{ Gastrointestinal system } \\
\hline Diarrhoea & $1(5)$ & $7(33)$ & $1(5)$ \\
\hline Upper abdominal pain & 0 & $3(14)$ & $1(5)$ \\
\hline Abdominal distension & 0 & $3(14)$ & $1(5)$ \\
\hline Abdominal pain & 0 & $3(14)$ & 0 \\
\hline Vomiting & 0 & $1(5)$ & $2(10)$ \\
\hline Nausea & 0 & $2(10)$ & 0 \\
\hline \multicolumn{4}{|l|}{ Nervous system } \\
\hline Headache & $7(32)$ & $6(29)$ & $7(33)$ \\
\hline Dizziness & $1(5)$ & $1(5)$ & $2(10)$ \\
\hline Paraesthesia & 0 & 0 & $2(10)$ \\
\hline \multicolumn{4}{|l|}{ Infections } \\
\hline Nasopharyngitis & 0 & $1(5)$ & $2(10)$ \\
\hline \multicolumn{4}{|l|}{ General } \\
\hline Fatigue & 0 & 0 & $2(10)$ \\
\hline \multicolumn{4}{|c|}{$\begin{array}{l}\text { Adverse events were monitored from day } 1 \text { to } 56 \text { of the study including follow-up period. Data are in } n \text { (\%). The listed } \\
\text { adverse events (any severity) have an incidence greater than one patient ( } 5 \% \text { ) in any treatment period. }\end{array}$} \\
\hline
\end{tabular}




\section{Figure 3}

Changes from baseline in itch intensity scores according to treatment period (A) $0-10$ numerical rating scale. (B) Primary biliary cholangitis-40 itch domain score. (C) 5-D itch scale. Error bars are $95 \%$ CI. LS=least squares.

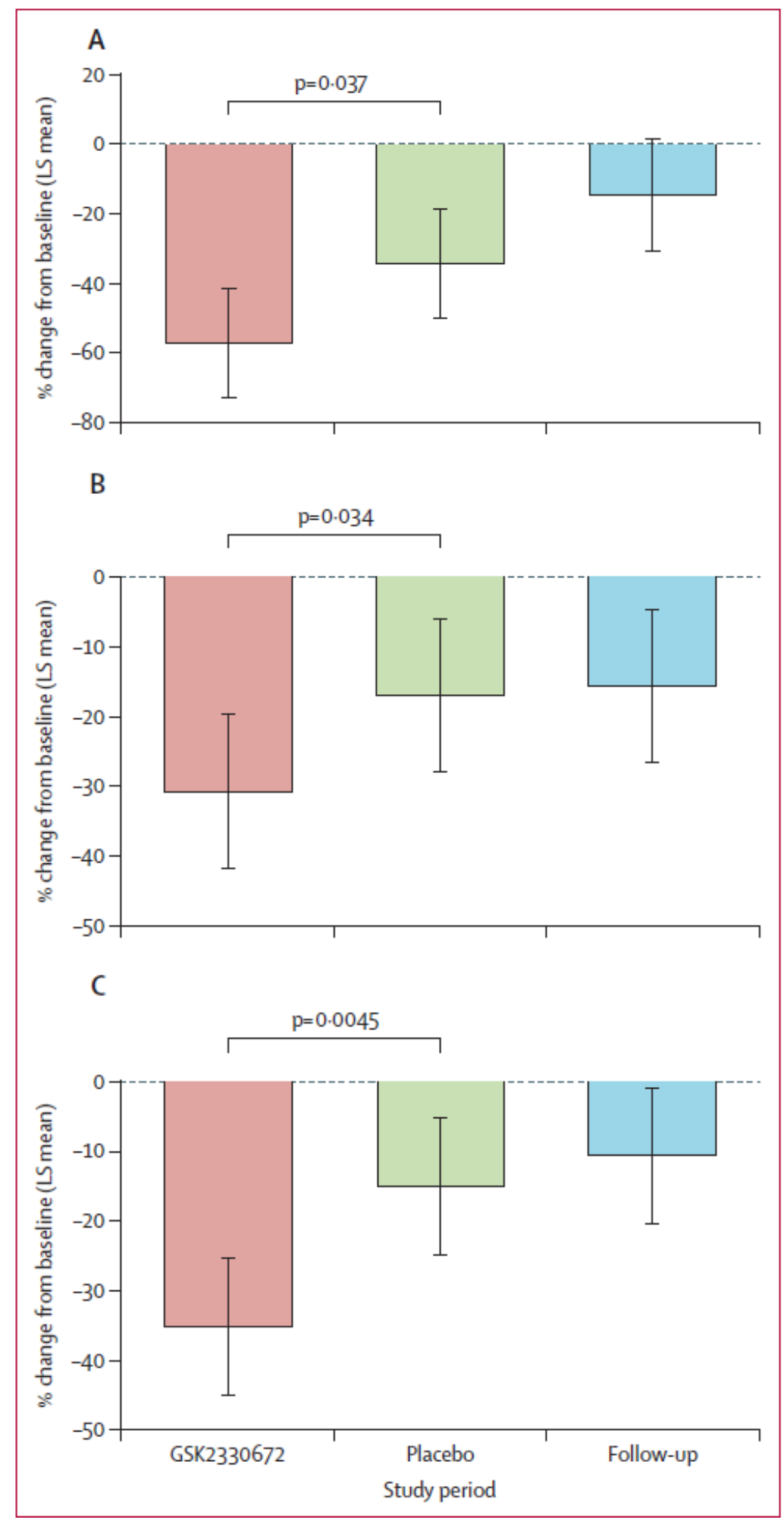




\section{Figure 4}

Changes in serum total bile acids and $\mathrm{C} 4$ according to treatment period. Following treatment with GSK2330672 a significant decrease in total bile acid (A) and significant increase in C4 (B) was seen. Data shown are geometric mean values and error bars show 95\% CI.

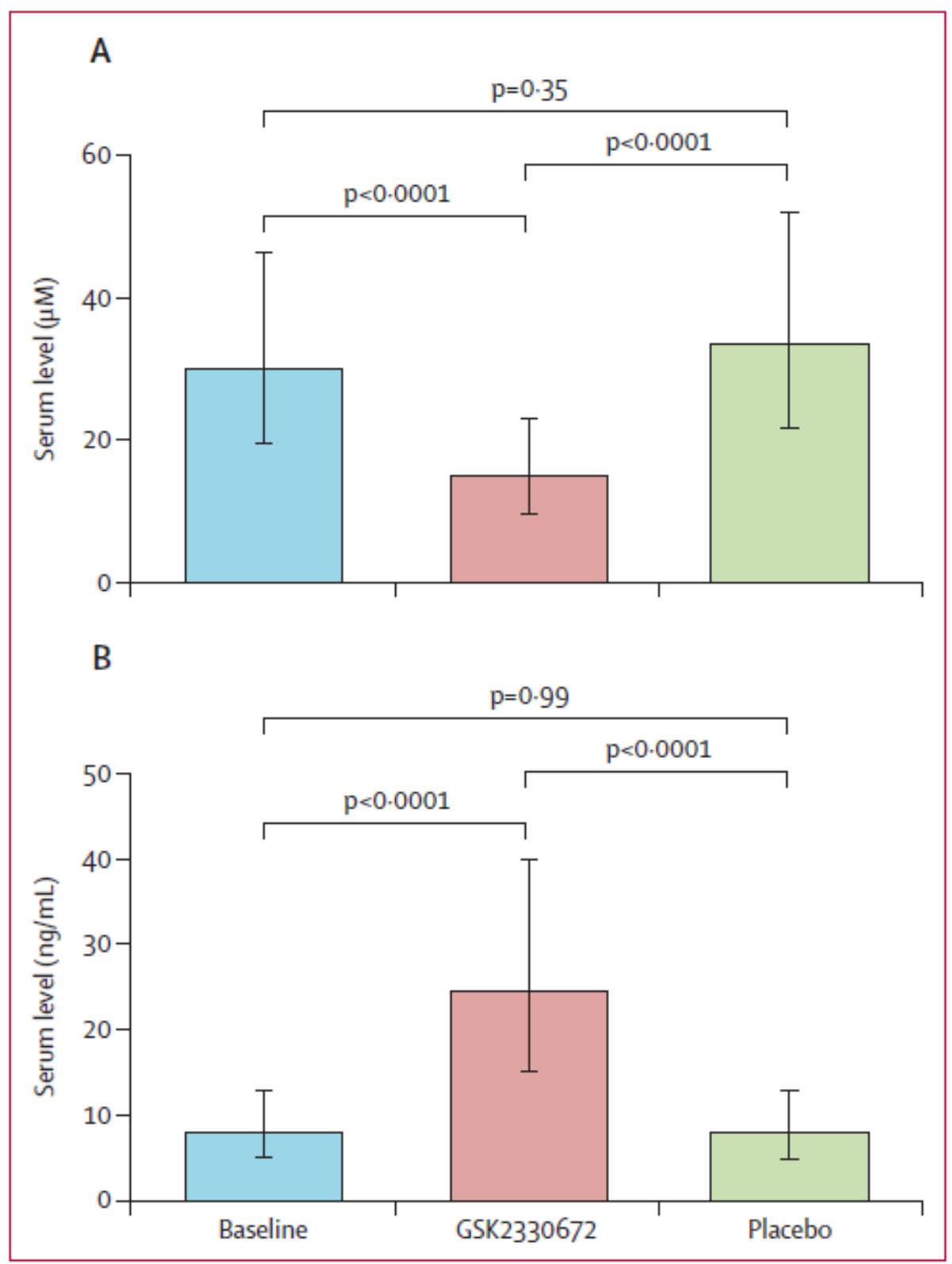




\section{References}

1 Beuers U, Gershwin ME, Gish RG, et al. Changing nomenclature for PBC: From 'cirrhosis' to 'cholangitis'. Hepatology 2015; 62: 1620-22.

2 Talwalkar JA, Souto E, Jorgensen RA, Lindor KD. Natural history of pruritus in primary biliary cirrhosis. Clin Gastroenterol Hepatol 2003; 1: 297-302.

3 Hegade VS, Mells GF, Lammert C, et al. A comparative study of pruritus in PBC cohorts from UK, USA and Italy. J Hepatol 2015; 62: S785.

4 Hegade VS, Mells GF, Beuers U, et al. Patient experience and characteristics of cholestatic pruritus in the UK-PBC research cohort. Hepatology 2014; 60: 339A-69A.

5 Beuers U, Kremer AE, Bolier R, Elferink RP. Pruritus in cholestasis: facts and fiction. Hepatol 2014; 60: 399-407.

6 Rudic JS, Poropat G, Krstic MN, Bjelakovic G, Gluud C. Ursodeoxycholic acid for primary biliary cirrhosis. Cochrane Database Syst Rev 2012; 12: CD000551.

7 Lindor KD, Gershwin ME, Poupon R, et al. Primary biliary cirrhosis. Hepatology 2009; 50: 291-308.

8 Beuers U, Boberg KM, Chapman RW, et al. EASL Clinical Practice Guidelines: management of cholestatic liver diseases. J Hepatol 2009; 51: 237-67.

9 Kuiper EM, van Erpecum KJ, Beuers U, et al. The potent bile acid sequestrant colesevelam is not effective in cholestatic pruritus: results of a double-blind, randomized, placebocontrolled trial. Hepatology 2010; 52: 1334-40.

10 Prince MI, Burt AD, Jones DE. Hepatitis and liver dysfunction with rifampicin therapy for pruritus in primary biliary cirrhosis. Gut 2002; 50: 436-39. 
11 Levy C. Management of pruritus in patients with cholestatic liver disease. Gastroenterol Hepatol 2011; 7: 615-17.

12 Hegade VS, Krawczyk M, Kremer AE, et al. The safety and efficacy of nasobiliary drainage in the treatment of refractory cholestatic pruritus: a multicentre European study. Alimentary Pharmacol Therapeut 2016; 43: 294-302.

13 Dyson JK, Webb G, Hirschfield GM, et al. Unmet clinical need in autoimmune liver diseases. J Hepatol 2015; 62: 208-18.

14 Hirschfield GM, Mason A, Luketic V, et al. Efficacy of obeticholic acid in patients with primary biliary cirrhosis and inadequate response to ursodeoxycholic acid. Gastroenterology 2015; 148: 751-61. e8.

15 Nevens F, Andreone P, Mazzella G, et al. A placebo-controlled trial of obeticholic acid in primary biliary cholangitis. $N$ Eng $J$ Med 2016; 375: 631-43.

16 Hegade VS, Speight RA, Etherington RE, Jones DE. Novel bile acid therapeutics for the treatment of chronic liver diseases. Therap Adv Gastroenterol 2016; 9: 376-91.

17 Dawson PA, Haywood J, Craddock AL, et al. Targeted deletion of the ileal bile acid transporter eliminates enterohepatic cycling of bile acids in mice. J Biol Chem 2003; 278: 33920-27.

18 Dawson PA, Lan T, Rao A. Bile acid transporters. J Lipid Res 2009; 50: 2340-57.

19 Hofmann AF. Inappropriate ileal conservation of bile acids in cholestatic liver disease: homeostasis gone awry. Gut 2003; 5: 1239-41. 
20 Lanzini A, De Tavonatti MG, Panarotto B, et al. Intestinal absorption of the bile acid analogue $75 \mathrm{Se}$-homocholic acid-taurine is increased in primary biliary cirrhosis, and reverts to normal during ursodeoxycholic acid administration. Gut 2003; 52: 1371-75.

21 Hofmann AF. Bile acids: trying to understand their chemistry and biology with the hope of helping patients. Hepatology 2009; 49: 1403-18.

22 Graff ner H, Gillberg PG, Rikner L, Marschall HU. The ileal bile acid transporter inhibitor A4250 decreases serum bile acids by interrupting the enterohepatic circulation. Alimentary Pharmacol Therapeut 2016; 43: 303-10.

23 Miethke AG, Zhang W, Simmons J, et al. Pharmacological inhibition of apical sodiumdependent bile acid transporter changes bile composition and blocks progression of sclerosing cholangitis in multidrug resistance 2 knockout mice. Hepatology 2016; 63: 512-23.

24 Baghdasaryan A, Fuchs CD, Osterreicher $\mathrm{CH}$, et al. Inhibition of intestinal bile acid absorption improves cholestatic liver and bile duct injury in a mouse model of sclerosing cholangitis. J Hepatol 2016; 64: 674-81.

25 Mayo MJ, Jones D, Bowlus C, et al. CLARITY: a phase 2, randomized, double-blind, placebo controlled study of lopixibat chloride (formerly LUM001), a novel apical sodiumdependent bile acid transporter inhibitor, in the treatment of primary biliary cirrhosis associated with itching. J Hepatol 2016; 64: S183-212.

$26 \mathrm{Wu}$ Y, Aquino CJ, Cowan DJ, et al. Discovery of a highly potent, nonabsorbable apical sodium-dependent bile acid transporter inhibitor (GSK2330672) for treatment of type 2 diabetes. J Med Chem 2013; 56: 5094-114. 
27 Nunez DJ, Yao X, Lin J, et al. Glucose and lipid eff ects of the ileal apical sodium-dependent bile acid transporter inhibitor GSK2330672: double-blind randomized trials with type 2 diabetes subjects taking metformin. Diabetes Obes Metab 2016; 18: 654-62.

28 Hegade VS, Kendrick SF, Dobbins RL, et al. BAT117213: ileal bile acid transporter (IBAT) inhibition as a treatment for pruritus in primary biliary cirrhosis: study protocol for a randomised controlled trial. BMC Gastroenterol 2016; 16: 71.

29 International Conference on Harmonisation (ICH). Harmonised tripartite guideline. Guideline for good clinical practice, Version 10; 1996.

30 Tandon P, Rowe BH, Vandermeer B, Bain VG. The effi cacy and safety of bile Acid binding agents, opioid antagonists, or rifampin in the treatment of cholestasis-associated pruritus. $\mathrm{Am}$ J Gastroenterol 2007; 102: 1528-36.

31 Khurana S, Singh P. Rifampin is safe for treatment of pruritus due to chronic cholestasis: a meta-analysis of prospective randomized-controlled trials. Liver Int 2006; 26: 943-48.

32 Alrefai WA, Saksena S, Tyagi S, Gill RK, Ramaswamy K, Dudeja PK. Taurodeoxycholate modulates apical Cl-/OH- exchange activity in Caco2 cells. Digest Dis Sci 2007; 52: 127078.

33 Raimondi F, Santoro P, Barone MV, et al. Bile acids modulate tight junction structure and barrier function of Caco-2 monolayers via EGFR activation. Am J Physiol Gastrointest Liver Physiol 2008; 294: G906-13.

34 Rao AS, Wong BS, Camilleri M, et al. Chenodeoxycholate in females with irritable bowel syndrome-constipation: a pharmacodynamics and pharmacogenetic analysis. Gastroenterol 2010; 139: 1549-58. 
35 Kremer AE, Feramisco J, Reeh PW, Beuers U, Oude Elferink RP. Receptors, cells and circuits involved in pruritus of systemic disorders. Biochima Biophys Acta 2014; 1842: 86992.

36 Alemi F, Kwon E, Poole DP, et al. The TGR5 receptor mediates bile acid-induced itch and analgesia. $J$ Clin Invest 2013; 123: 1513-30.

37 Lieu T, Jayaweera G, Zhao P, et al. The bile acid receptor TGR5 activates the TRPA1 channel to induce itch in mice. Gastroenterology 2014; 147: 1417-28.

38 Sauter G, Berr F, Beuers U, Fischer S, Paumgartner G. Serum concentrations of 7alphahydroxy-4-cholesten-3-one reflect bile acid synthesis in humans. Hepatology 1996; 24: 12326.

39 Kremer AE, van Dijk R, Leckie P, et al. Serum autotaxin is increased in pruritus of cholestasis, but not of other origin, and responds to therapeutic interventions. Hepatology 2012;

56: $1391-400$.

40 Kremer AE, Martens JJ, Kulik W, et al. Lysophosphatidic acid is a potential mediator of cholestatic pruritus. Gastroenterology 2010;139: 1008-18.

41 Jones DE. Pathogenesis of cholestatic itch: old questions, new answers, and future opportunities. Hepatology 2012; 56: 1194-96 